\title{
Simulation Performance of CDMA/QA in Mobile Channel
}

\author{
Khalid Hamid Bilal Abdalla ${ }^{1}$, PhD.
}

\section{Abstract}

The aim of this work is to study, analyze and evaluate software the link performance of mobile communication system under the impairment of the multiple access schemes, down-link thermal noise and the multipath fading in the mobile channel.

For this purpose, modeling and simulation has been used to study the effects of nonlinearities in mobile channel using spread spectrum for Direct Sequence Code Division Multiple Access, with Quadrature Amplitude Modulation (DSCDMA/QAM).

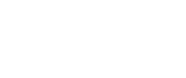

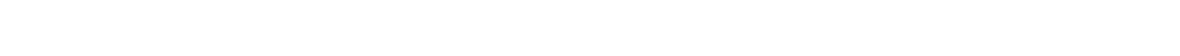

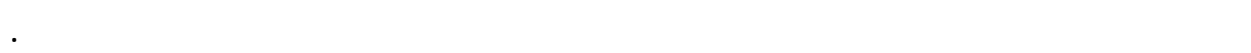

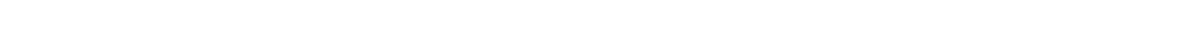

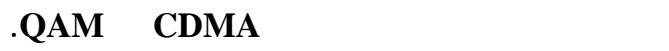

\footnotetext{
${ }^{1}$ Omdurman Islamic University
} 


\section{Introduction}

Spread spectrum modulation, is a wireless communication technique, uses a transmission bandwidth many times greater than the information bandwidth or data rate of any user [1]. There are many types of spread spectrum techniques such as Direct Sequence (DS), Frequency Hopping (FH), and Time Hopping (TH), a hybrid or a combination of these methods [2]. The spectrum spreading in DS is measured by the processing gain (Gp) in decibels [3]. Direct Sequence based Code Division Multiple Accesses (DS-CDMA) has taken on a significant role in the proposed land mobile satellite, cellular and personal communications systems. Direct sequence code division multiple access (DS-CDMA) systems are, at present, looking very promising compared to the conventional advanced frequency division multiple access (FDMA) and time division multiple access (TDMA) systems. Some of these advantages are in $[4,5,6]$. Recently there has been increasing interest in using direct sequence spread spectrum (DSSS) code division multiple accesses (CDMA) for commercial applications. DS-CDMA is proposed for satellite [7] and for indoor/terrestrial [8, 9].

\section{Analysis}

The system model is depicted in Fig 1.

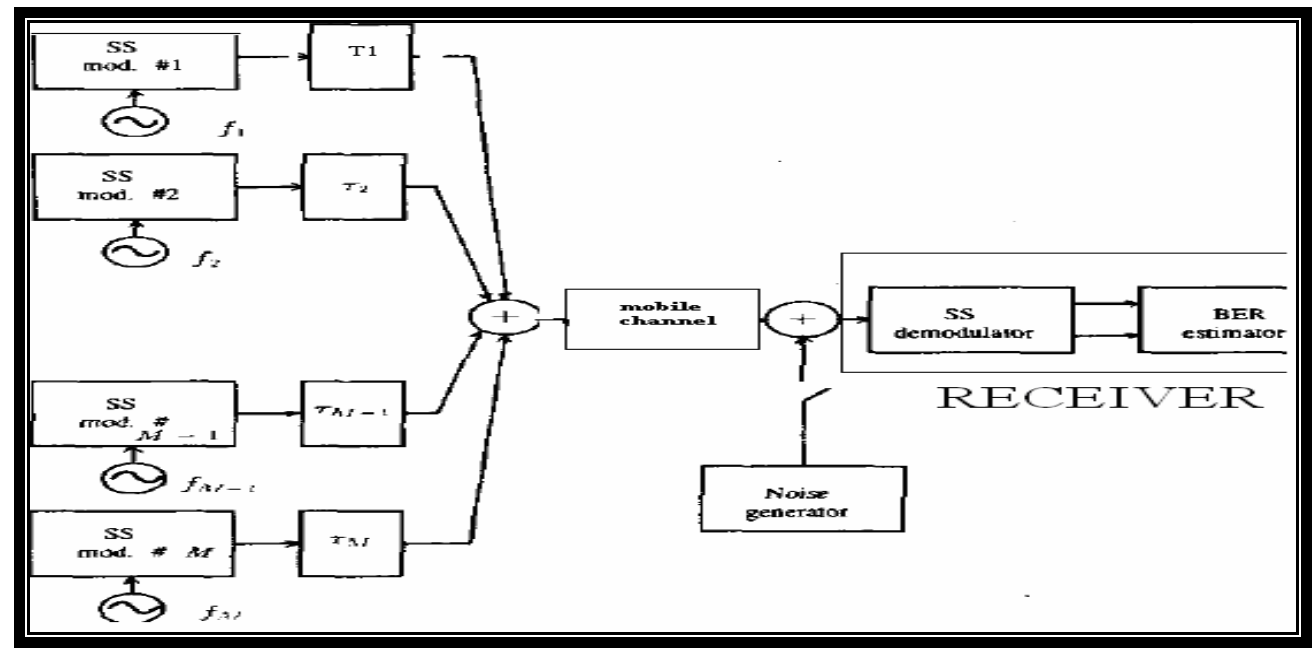

Figure (1) DS- CDMA system model 
In DS-CDMA, users employ their own sequence to spread the information data. The information data of each user is modulated by QAM then the first bits of modulated data is spreaded by the code sequence and the spreaded data of all users are transmitted to the base station at the same time. The base station detects the information data of each user by correlating the received signal with the code sequence allocated to each user. The transmitted data in the in-phase channel and quadrature phase modulated by QAM are multiplied by the code sequence used to spread the transmitted data then the transmitted signal is contaminated in a Rayleigh fading channel .[10].

At the receiver, AWGN is added to the received data, the resampled data are the data of all users. By correlating the data with the spread code used at the transmitter, the transmitted data of all users is detected.

In this model each user sends a QAM signal with inphase (I) and qudrature $(\mathrm{Q})$ components spread by the respective user code sequence with high power. Analytically, The general form of an QAM signal can be defined as: [16].

$$
\begin{gathered}
S_{i}=\sqrt{\frac{2 E_{\min }}{T_{S}}} a_{i} \cos \left(2 \pi f_{c} t\right)+\sqrt{\frac{2 E_{\min }}{T_{S}}} b_{i} \sin \left(2 \pi f_{c} t\right) \\
0 \leq t \leq T, i=1,2, \ldots, M
\end{gathered}
$$

Where $E_{\min }$ is the energy of the signal with the lowest amplitude and $a_{i}$ and $b_{i}$ are pair of independent integers chosen according to the location of the particular signal point.

The continuous time representation of the equivalent base band DSCDMA signal is given by: [18] 


$$
\begin{aligned}
& S_{D S}(t)=\sum_{u=1}^{N_{u}} \sum_{m=-\infty}^{\infty} \sum_{n=0}^{N-1} D_{m}^{u} c_{n}^{u} g_{n}(t-m T) \\
& g_{n}(t)=\left(\begin{array}{cc}
e^{j 2 \pi n(t-T c)} & t \in[0, T] \\
0 & t \notin[0, T]
\end{array}\right)
\end{aligned}
$$

Where $g_{n}(t-m T)$ represents the $n$th sub carrier, $T$ is the duration of the $\mathrm{MC}$ CDMA symbol and $\mathrm{T}_{\mathrm{cp}}$ is the duration of the cyclic prefix. The signal is low pass filtered before transmission.

where $\mathrm{D}_{\mathrm{mn}}{ }^{\mathrm{u}}$ is the $\mathrm{S} / \mathrm{P}$ output for time slot $\mathrm{m}$, sub carrier $\mathrm{n}$ and user $\mathrm{u}, \mathrm{c}(\mathrm{t})$ is the WH code for user $u$ and $g_{n}(t-m T)$ is the $n$th sub carrier (as defined in [2]. The average probability of error $\mathrm{P}_{\mathrm{p}}$ for in-phase branch can be expressed as [17].

$$
\mathrm{p}_{\mathrm{p}}=\frac{1}{2}\left[\mathrm{P}_{1}\left\{s_{p}>\left.0\right|_{d p}=-1\right\} \mathrm{p}_{\mathrm{r}}\left\{s_{p}<\left.0\right|_{d_{p}}=+1\right\}\right]
$$

Assuming that the probabilities of transmitting symbols -1 and +1 are equal (i.e.):

$$
\mathrm{P}_{\mathrm{p}}=\mathrm{P}_{\mathrm{r}}\left\{S_{p}<\left.0\right|_{\mathrm{d}_{\mathrm{p}}}=+1\right\}
$$

Assuming that the number of chip per bit, $\mathrm{N}$ is large, the decision variable $s_{p}$ can be approximated according to the central limit theorem by a Gaussian random variable. The error probability in an AWGN channel for QAM using coherent detection can be approximated by: [16] 
$\mathrm{p}_{\mathrm{ep}}=4\left(1-\frac{1}{\sqrt{L}}\right) Q\left(\sqrt{\frac{\left[3 E\left(s_{p}^{1}(r)\right)\right]^{2}}{(L-1) \operatorname{var}\left(s_{p}^{1}(r)\right)}}\right)$

And for QAM implies that $\mathrm{L}=4$ and

$\mathrm{p}_{\mathrm{ep}}=2 Q\left(\sqrt{\frac{\left[3 E\left(s_{p}^{1}(r)\right)\right]^{2}}{15\left(\operatorname{var}\left(s_{p}^{1}(r)\right)\right.}}\right)$

$\mathrm{E}\left(\mathrm{s}_{\mathrm{p}}^{1}(1)\right)$ Is the mean and $\operatorname{var}\left(s_{p}^{1}(r)\right)$ is the variance of the decision variable $s_{p}^{1}$. The treatment for the quadrature branch is similar to in-phase branch.

Therefore:

$\mathrm{p}_{\mathrm{eq}}=2 Q\left(\sqrt{\frac{\left[3 E\left(s_{q}^{1}(r)\right)\right]^{2}}{15 \operatorname{var}\left(s_{q}^{1}(r)\right)}}\right)$

The total error probability can then be written as:

$\mathrm{p}_{\mathrm{e}}=2 Q\left(\sqrt{\frac{3 \mu_{1}^{2}}{15 \sigma_{1}^{2}}}\right)+2 Q\left[\sqrt{\frac{3 \mu_{2}^{2}}{15 \sigma_{2}^{2}}}\right]$

Where the mean $\mu_{1}, \mu_{2}$ and the variance $\sigma_{1}^{2}, \sigma_{2}^{2}$

\section{Principle of Monte Carlo Simulation}

The Monte Carlo simulation is a one-to-one correspondence with the real system within the limits of modeling assumptions and approximations. 
The expected value $E\{g(Y(t))$ is estimated from MC simulations according to: $[11]$

$$
E\left\{g\left(Y^{\Lambda}(t)\right\}=\frac{1}{N} \sum_{i=1}^{N} g(Y(i)\right.
$$

Where the caret indicates estimated value and $N$ is the number of samples simulated. Monte Carlo simulation for estimating the bit error rate in mobile communication system is shown in fig. (2) that involves the following steps: 1-The signal is generated by form Inphase (I) and Quadrature (Q) data streams for all users for DS-CDMA/QAM.

2- Then the output signal encounters the degrading effects of the propagation channel.

3- Channel type is modeled to represent environmental conditions of Rayleigh channel.

4- Before detection of the signal, it is subjected to the effect of the Additive Gaussian noise (AWGN).

5- The detection technique is implemented where despreading sequence signal is assumed after demodulation. Finally the decision and the estimation of error rate are performed as:

$$
P_{e}^{\Lambda}=\frac{1}{N} \sum_{k=1}^{N} g(Y(k))
$$

where $g(Y(k))=1$ if $\mathrm{Y}(\mathrm{k}) \neq \mathrm{A}(\mathrm{k})$ and $g(Y(k))=0$ if $Y(k)=A(k)$ (this step is equivalent to counting errors).

The accuracy of estimates obtained via MC simulation will depend on the estimation procedure used, sample size $N$, the ability to reproduce sampled values of the input processes accurately, and modeling assumptions 
and approximations. In general, the accuracy will be proportional to large number of samples will have to be simulated in order to obtain accurate estimates via $\mathrm{MC}$ simulations. While the $\mathrm{MC}$ technique is general and can be applied to a broad range of problems, the large sample size requirements and hence the length of simulation are often limiting factors.

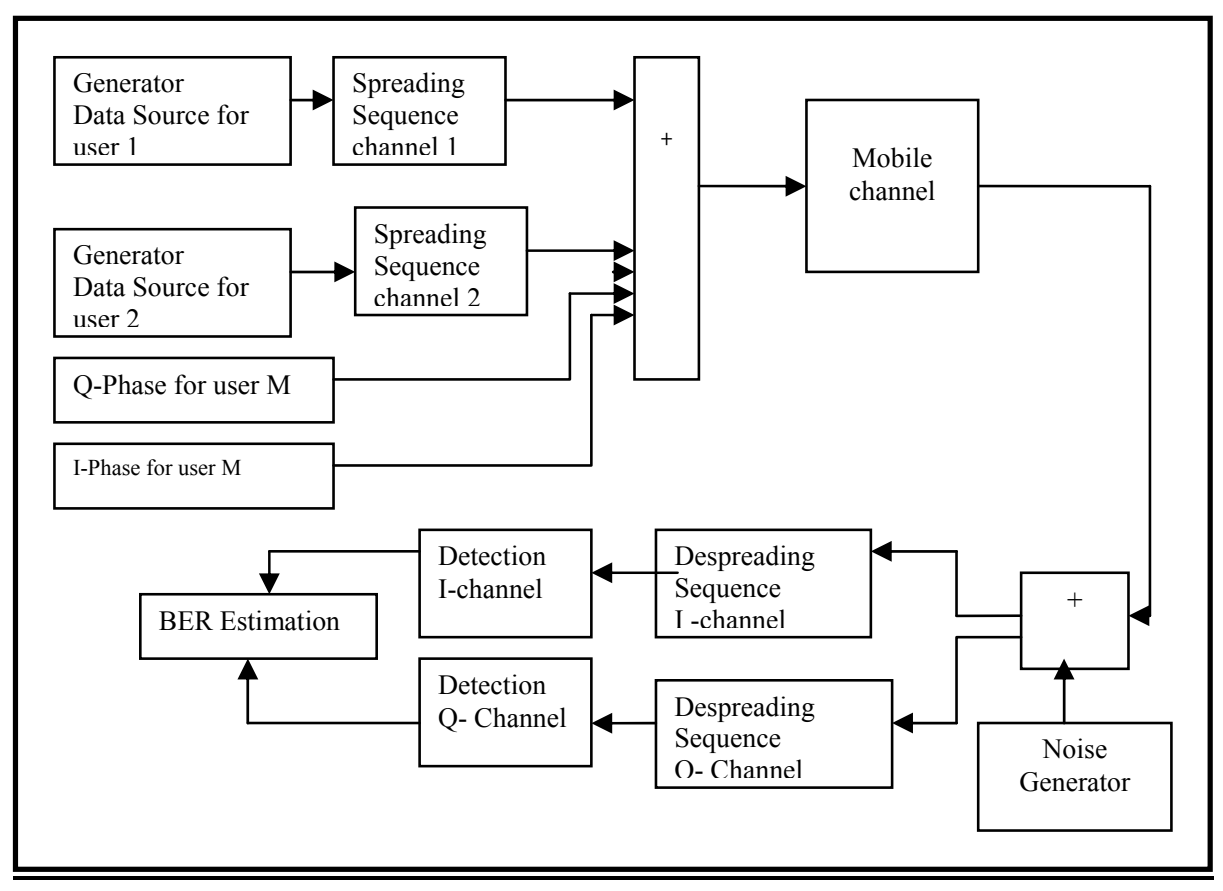

Figure 2.Monte Carlo Simulation Model of a Mobile Channel [5]

According to the Central Limit Theorem [15], Gaussian noise generator, can represent the up-down-link-noise, having zero mean $\left(\mathrm{U}_{0}\right)$ and variance $\left(\sigma^{2}\right)$ which depends on the signal-to-noise ratio $(S / N)$ in down links. Urban areas are characterized by almost complete obstruction of the line-of-sight component of the received signal. The received envelope is found to be Rayleigh distributed and the phase having uniform statistical 
distributed in the range $(0-2 \pi)$. The signal mainly propagates by way of scattering. Each wave experiences a Doppler frequency shift given by [12].

$$
\mathrm{f}_{\mathrm{m}}=\left(\mathrm{v} / \lambda_{1}\right) \cos (\theta)
$$

Where $\mathrm{v}=60 \mathrm{~km} / \mathrm{h}$ is the assumed vehicle speed, $\lambda_{1}=\left(\mathrm{c} / \mathrm{f}_{0}\right)$ is the carrier wavelength where $\mathrm{c}$ is the speed of light $\left(3 \times 10^{8} \mathrm{~m} / \mathrm{sec}\right)$ and $\mathrm{f}_{0}$ is the carrier frequency assumed $(1.5 \mathrm{GHz}), \theta$ is the phase difference between the incident wave and the direction of vehicle motion.

The Rayleigh model is given by $[13,14]$

$$
\begin{aligned}
& \mathrm{T}_{\mathrm{c}}(t)=2 \sum_{n=1}^{N_{0}} \cos \left(\beta_{n}\right) \cos \left(\omega_{n} t\right)+\sqrt{2} \cos \left(\omega_{m} t\right) \cos (\alpha) \\
& \mathrm{T}_{\mathrm{s}}(t)=2 \sum_{n=1}^{N_{0}} \sin \left(\beta_{n}\right) \cos \left(\omega_{n} t\right)+\sqrt{2} \sin \left(\omega_{m} t\right) \sin (\alpha)
\end{aligned}
$$

Where:

$$
\beta_{n}=\pi n / N_{0} \omega_{n}=\omega_{m} \cos \left(2 \pi n / N_{0}\right), \omega_{m}=2 \pi V / \lambda_{1}, N_{0}=0.5[0.5 N-1], N=34
$$

\section{Planning}

A flow chart representing all steps that taken from the data source to the received data to simulate DS-CDMA system is shown in fig. 3 below. Each section of the flow chart represents a functional component of signal generation. The flow chart shows the transmission model used by Matlab program to simulate DS-CDMA system. Flow chart illustrates the signal flow in the program. 


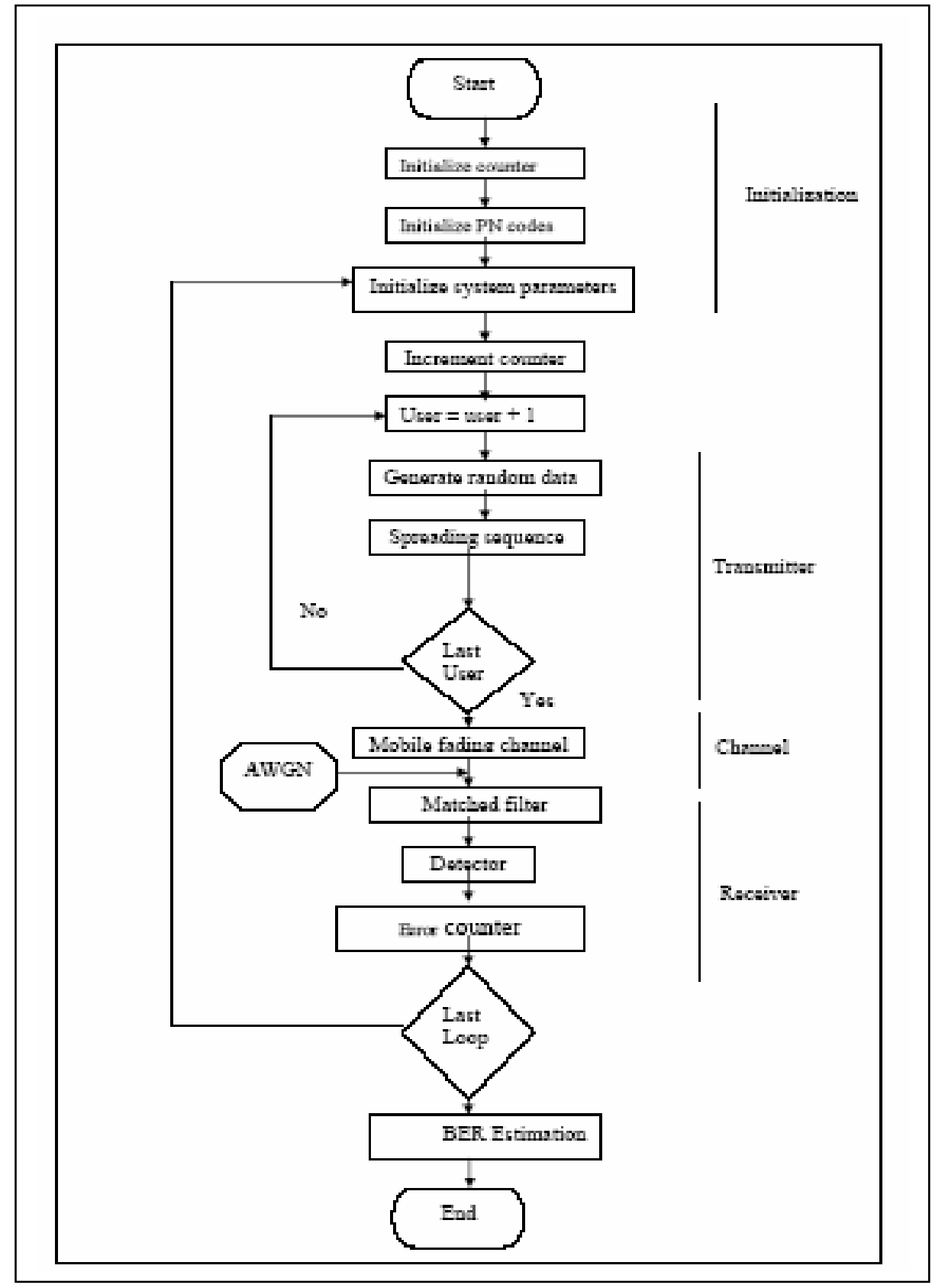

Fig. 3 Flow chart 
A computer program using Matlab is implemented to simulate the BER performance in a AWGN and Rayleigh fading environments of DSCDMA [8]

\section{Simulation Results :}

A matlab program is executed for estimating the bit error rate at different values of the control parameters (signal to noise ratio SNR and propagation channel).

The performance results are plotted and analyzed for multi-user CDMA/QAM in mobile channel for different cases.

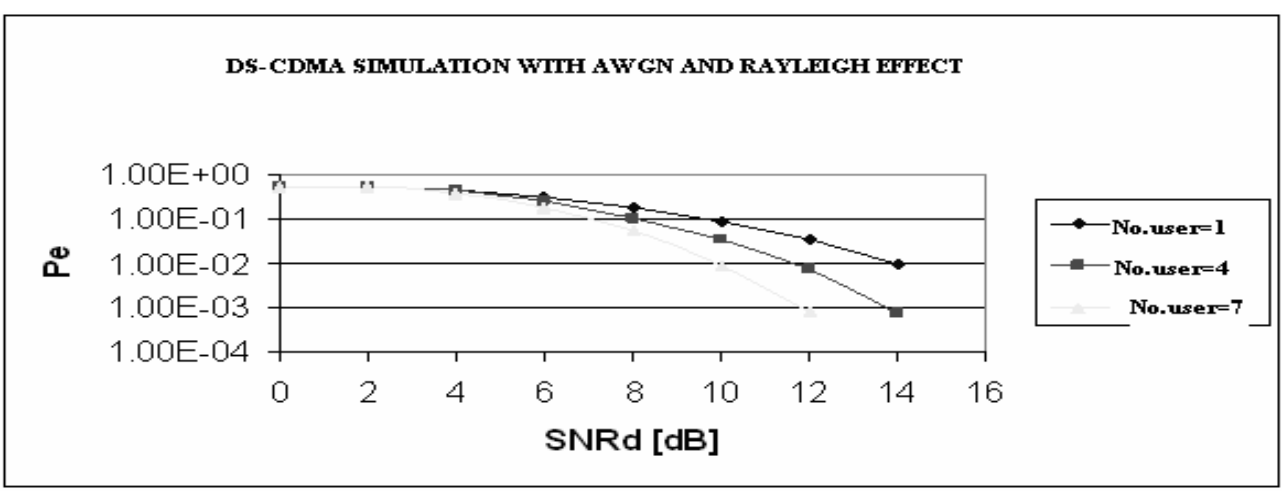

Figure 4. Probability of Error Performance over a Rayleigh Channel for Different Number of Users 
DS-CDMLA/QAM STMULATION WITH AWGN AND RAYLEIGH FADING

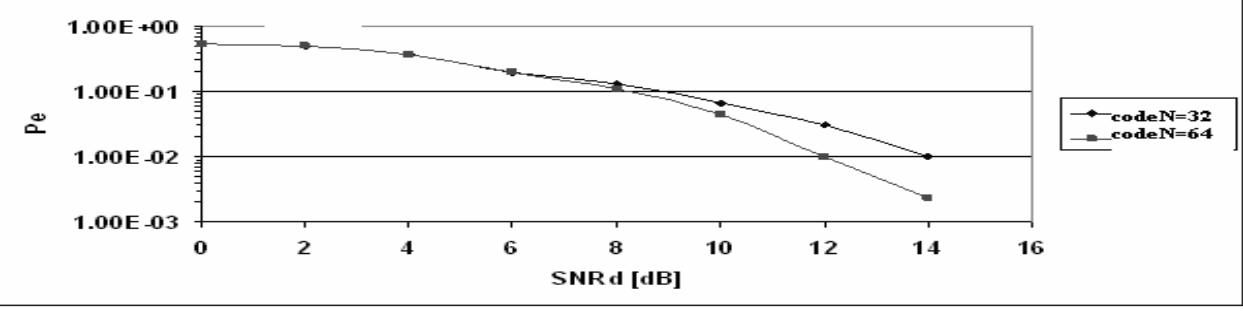

Figure 5 Performance of Rayleigh Channel, Comparison between Different Code Lengths

DS-CDMA/QAM COMPARSION BETWEEN SIMULATION AND ANALYTICAL

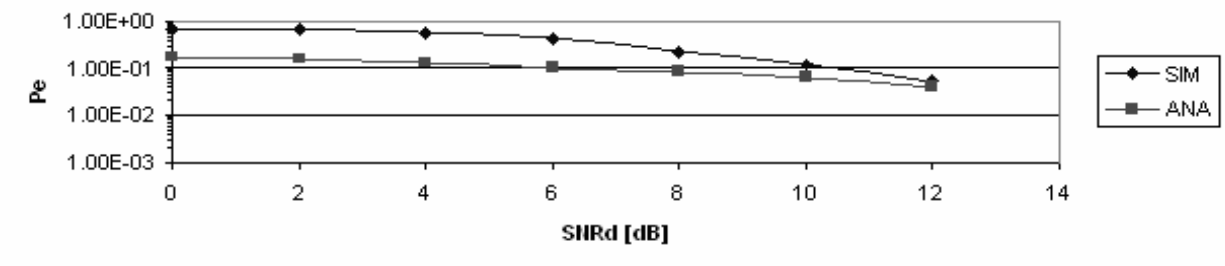

Fig. 6 Comparison Between Analytical and Simulation Performance for

7 Number of User

DS-CDMLA/QAM COMPARISON BETWEEN SMMULATION AND ANALTICAL FOR ONE USER

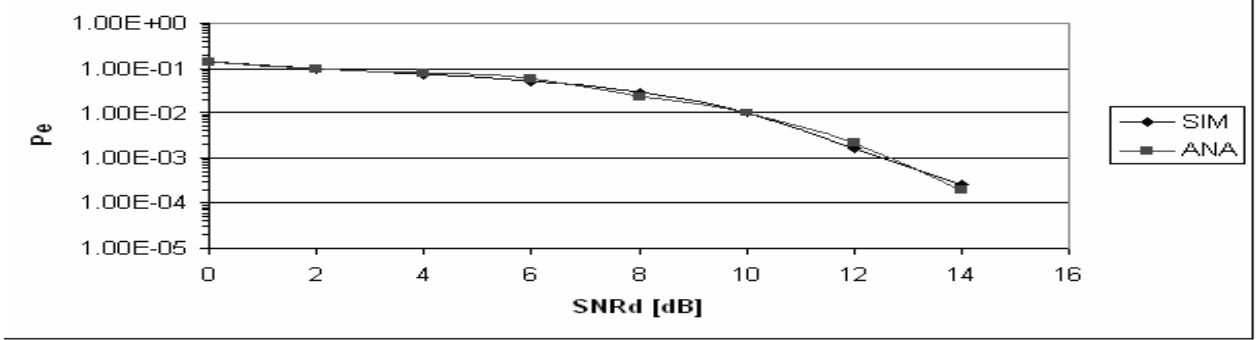

Fig 7. Comparison between Analytical and Simulated results For Rayleigh Channel Performance for One User 


\section{Conclusions}

In this paper, the performance of DS-CDMA signal transmission through mobile channels system with QAM mapping in the presence of AWGN and multipath fading using computer simulation model was studied, modeled, simulated and analyzed it is performance in terms of the BER was obtained.

The results obtained from simulation models show that:

- Increasing the signal to noise ratio reduces the value of Bit Error Rate (BER).

- Increasing the code length reduces the value of bit error rate up to a certain limit.

- Also, increasing the number of users increase the value of bit error rate to a certain limit depending on the SNR.

- Increasing the number of users (M), the system performance degrades because (MAI) dominate.

- Increasing the SNR improves the performance up to a certain limit after which any further increase does not have any significant effect on the quality of the received signal.

\section{References:}

[1] A.J. Viterbi, CDMA: Principles of spread spectrum communications, Addison-Wesley, 1995.

[2] R. Prasad“"CDMA for wireless personal communications," Artech House, Boston. London, 1996

[3] B. Y. Chung. C. Chien. H. Samueli, and R. Jam, "Performance an of an $\mathrm{M}$ array BPSK direct sequence spread-spectrum receiver architecture IEEE. 
Journal on Selected Areas in comm. vol. 11. No. 7, pp. 1 ،1107-096Sept 1993.

[4] R. F. Ormondroyd and J. J. Maxey,"Performance of low-rate orthogonal convolutional codes in DS-CDMA applications," IEEE Trans. Veb. Tech. pp320-328. May 1997.

[5] I Glover and P. Grant, Digital communications," Prentice Hall Europe, 2003.

[6] S. Glisic and B. Vucetic, "Spread spectrum CDMA system for wireless communications," Artech House, Inc. 2005.

[7] R. De Gaudenzi, C. Elia, and R. Viola, "Bandlimited quasisynchronous. CDMA: A novel satellite access technique for mobile and personal communication systems". IEEE 1. Select. Areas Commun., vol. 10, no. 2, pp. 328-343, Feb. 1992

[8] W. C. Y. Lee, "Overview of cellular CDMA)", IEEE Tans. Veh. Technol, vol. 40, NO.2, pp.291-302, May 1991.

[9] K. S. Gilhousen, 1. M. Jacobs, R. Padovani, A. J. Viterbi, L. A. Weaver, and C. E. Wheatley, "On the capacity of a cellular CDMA system," IEEE Trans. Veh. Teclmol., vol. 40, pp.303-3 12, May 1991

[10] Logsdon, "Mobile communication satellites, theory and applications," McGraw-Hill, Inc. 1995.

[11] E. Lutz, D. Cygan, M. Dippold, F. Dolainsky, and W. Papke, "The land mobile satellite communication channel-recording, statistics, and channel model," IEEE Trans. on Veh. Tech. Vol. 40, No. 2, pp 375 - 86 May. 1991. 
[12] J.P. Castro," Statistical observations of data transmission over land mobile satellite

Channel” . IEEE. Journal on selected Areas in comm, vol. 10 No.8,pp.12271235,oct1992.

[13]W.C.Jackes,Jr,” Microwave mobile communication “John wily\&Sons. 1974.

[14]D.Parsons, "The mobile radio propagation channel" Pentech Press ,London 1992.

[15] A. M. Law and W.D. Kelton, "Simulation modeling \& analysis" Me Graw-Hall, In. 1991.

[16] Refaat El-Zanfally, "Performance Analysis of 16-ARAY QAM Systems in Nonlinear Satellite Channels, Ph.D. Dissertation, MTC, Cairo, Egypt, 1988.

[18] Kh. Ben Lataief, "Efficient evaluation of the error probabilities of spread spectrum multiple access communication” IEEE Trans. Commum., vol. 45, No. 2, pp. 239 - 246, Feb 1997.

[18] M. Mayhoub, R. El Zanfally, H. El Hennawy, and S. Mahrous, "Performance analysis of synchronous DS-CDMA in mobile nonlinear satellite channel”, Fiftenth National Radio Science Conf. Helwan, Cairo, Egypt, (C12), PP. 1 - 14, Feb. 1998.

[19] B. Vucetic and Jun Du, "Channel modeling and simulation in satellite mobile communication systems". IEEE. Journal on Selected Areas in comm, vol. 10, No.8, pp. 1209 -1218, Oct 1992. 